\title{
L'évolution moléculaire
}

La théorie la plus généralement admise de l'évolution moléculaire est celle des mutations neutres. De cette théorie découlent deux conséquences. Les mutations "synonymes ", qui portent sur une base sans entraîner de changement dans l'acide aminé codé, doivent être plus fréquentes que celles qui entraînent un tel changement elles le sont en moyenne d'un facteur 2 à 3 ; et lorsqu'un changement de séquence est sans effet sur l'activité biologique, comme dans les pseudogènes, la proportion des mutations non synonymiques doit augmenter. Par conséquent, en principe, plus une séquence est importante pour la fonction, moins elle doit évoluer. Il existe pourtant des exceptions frappantes à ce principe.

La mieux connue est celle des inhibiteurs des protéases à sérine, dont font partie l'alpha-1-antitrypsine et l'antithrombine (cf. $\mathrm{m} / \mathrm{s}$ $n^{\circ} 2$, vol. 1, p. 106). Ils possèdent un centre actif qui réagit avec le site actif de la protéase. Ce centre contient un acide aminé dit PI qui oriente l'activité, et dont le remplacement altère souvent la spécificité. Deux articles récents ont démontré que l'évolution moléculaire du centre actif se fait dans certains cas à une vitesse surprenante.

Hill et Hastie [1] (Edimbourg) ont isolé des $\mathrm{ADN}$ complémentaires et des messagers de rongeurs qui codent pour des protéines voisines d'inhibiteurs connus : la "contrapsine ", inhibiteur chez la souris des protéases de type trypsine, et une protéine humaine qui lui ressemble mais qui a acquis une spécificité différente, l'alpha-1-antichymotrypsine. L'analyse des séquences protéiques connues ou déduites a conduit les auteurs à les diviser en trois domaines : alors que la presque totalité de la séquence présente des homologies très fortes entre les quatre protéines, la vingtaine d'acides aminés qui constituent le centre actif $\mathrm{m} / \mathrm{s} n^{\circ} 6$ vol. 3 , juin 87 (domaine 2) n'en dévoile aucune. Non seulement les mutations ont été très nombreuses, mais la proportion de celles qui ne sont pas synonymiques atteint et même dépasse celle des mutations synonymiques. La vitesse de substitution peut être évaluée comme étant 6 à 8 fois supérieure à celle des pseudogènes. Une conclusion analogue ressort d'un travail de Laskowski et al. (West-Lafayette, USA) qui ont séquencé le domaine 3 de l'ovomucoïde à partir de 100 espèces d'oiseaux [2]. La zone de contact inhibiteurprotéine est extrêmement variable alors que la conservation du reste de la molécule est bien plus grande, et le nombre des acides aminés qu'on peut trouver au site PI est au moins de 5.

Comment peut-on rendre compte des taux élevés de mutation observés dans le centre actif de ces molécules? L'hypothèse la plus probable est que ces mutations sont fixées par une sélection darwinienne positive. Il est cependant improbable que ces inhibiteurs agissent seulement sur des protéases endogènes, ce qui impliquerait une coévolution des protéases cibles. Hill et Hastie pensent qu'il s'agit d'adaptations à des protéases exogènes provenant d'agents infectieux ou de parasites, dont on sait qu'ils utilisent de telles protéases comme facteurs de virulence accrue. Comme les parasites peuvent modifier certaines de leurs protéines, une " course à l'évolution " [3] s'engagerait alors entre l'hôte et l'agent d'infestation.

Un autre aspect de l'évolution est illustré par $\mathrm{Li}$ et Tanimura [4] (Houston, USA), qui apportent des données nouvelles sur la vitesse de l'évolution moléculaire en fonction des espèces. S'effectue-t-elle à vitesse constante ou non? Cette question, déjà ancienne, est testée par des méthodes immunologiques, l'analyse des séquences de protéi- nes et maintenant d'ADN. Les auteurs étudient les vitesses de substitution de codons synonymes. Ils montrent que cette vitesse est de 4 à 10 fois plus élevée chez les rongeurs que chez les primates supérieurs, et 2 à 4 fois plus que chez les " artiodactyles ", nommément bœuf et chèvre. De plus l'évolution moléculaire s'est ralentie de façon significative dans la lignée hominoïde par rapport à celle de l'ensemble des singes et même des singes supérieurs.

Deux théories tentent d'expliquer les raisons de ces différences. A la théorie classique de la variation de durée des générations, qui conduit à une différence du nombre de réplications de l'ADN germinal par année, s'est opposée récemment la théorie d'une différence des modes de réparation de l'ADN selon les espèces. Pour Li et Tanimura, même s'il est possible que la réparation de l'ADN soit plus efficace chez l'homme, cette théorie n'est pas nécessaire : la durée d'une génération est 100 fois plus courte chez le rat que chez l'homme, et l'on calcule que le nombre de réplications de l'ADN germinal par an devrait être 10 fois plus élevé chez les rongeurs. La différence, bien que moindre, persiste chez les ruminants, les singes et même les primates non humains. On peut en tous cas conclure avec les auteurs que l'horloge moléculaire tourne moins vite pour l'homme que pour les singes et les autres primates.

J.-C. D.

1. Hill RE, Hastie ND. Accelerated evolution in the reactive centre regions of serine protease inhibitors. Nature 1987 ; 326 : 96-9.

2. Laskowski M, Kato I, Ardelt W, et al. Ovomucoid third domain from 100 avian species : isolation, sequences, and hypervariability of enzyme-inhibitor contact residues. Biochemistry $1987 ; 26: 202-21$.

3. Brown AL. Positively darwinian molecules ? Nature $1987 ; 326: 12-13$.

4. Wu WH, Tanimura M. The molecular clock runs more slowly in man than in apes and monkeys. Nature 1987 ; 326 : 93-6.

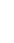

